\title{
Vom Ododion zum Smeller 2.0
}

Die literarisch-technische Erfindung der ,Duftorgel ${ }^{\star}$

De l'Ododion au Smeller 2.0. L'invention littéraire et technique de «l'orgue olfactif»

From the Ododion to the Smeller 2.0. The Literary and Technical Invention of the 'Perfume Organ'

\section{Sergej Rickenbacher}

\section{OpenEdition \\ Journals}

Édition électronique

URL : http://journals.openedition.org/ceg/3278

DOI : $10.4000 /$ ceg. 3278

ISSN : 2605-8359

Éditeur

Presses Universitaires de Provence

Édition imprimée

Date de publication : 15 novembre 2018

Pagination : 23-38

ISBN : 979-10-320-0183-7

ISSN : 0751-4239

\section{Référence électronique}

Sergej Rickenbacher, « Vom Ododion zum Smeller 2.0 », Cahiers d'Études Germaniques [Online], 75

2018, Online erschienen am: 25 April 2020, abgerufen am 27 November 2020. URL : http://

journals.openedition.org/ceg/3278; DOI : https://doi.org/10.4000/ceg.3278 


\title{
Vom Ododion zum Smeller 2.0 \\ Die literarisch-technische Erfindung der ,Duftorgel
}

\author{
Sergej RICKENBACHER \\ RWTH Aachen
}

\section{Literatur der Zukunft | Zukunft der Technik}

Literatur ist zuweilen nicht nur in ästhetischer Hinsicht der Zeit voraus. Vielmehr gehört die Prognose der Zukunft zum Auftrag der neuzeitlichen Fiktion. ${ }^{1}$ Im Sinne dieser Funktion entwickelt sich die Literatur seit der Industrialisierung im 19. Jahrhundert zu einem bevorzugten Medium, um zum einen Wissen über Technik $^{2} \mathrm{zu}$ vermitteln ${ }^{3}$ sowie zum anderen die Gefahren und Chancen eines zukünftigen maschinellen Zeitalters zu simulieren - nicht zuletzt durch die Imagination von technischen Objekten. Die Funktion der Literatur beschränkt sich aber nicht nur auf das Vermitteln und Entwerfen von Fortschritten, sondern produziert ein Wissen, das nicht immer mit dem mechanischen Dispositiv der Technik kohärent ist, und transformiert somit ebenfalls den Fortschritts- und Wissensbegriff. ${ }^{4}$ Auch wirkte die Literatur wieder auf die Technik zurück. Dass Texte und ihr Wissen die Vorstellungskraft der künftigen Ingenieure und Wissenschaftler beeinflussten, dürfte kaum bestritten werden: Welcher Ingenieur kannte nicht die Mondreisen von Jules Verne? ${ }^{5}$

Dieses Verhältnis zwischen Literatur, Wissenschaft und Technik sowie die prognostische Funktion fiktiver Texte blieben der Literatur- und

1. Vgl. Philipp Theisohn, Die kommende Dichtung. Geschichte des literarischen Orakels 1450-2050, München, Wilhelm Fink, 2012, bes. VII-IX.

2. Im Wissen, dass der griechische Begriff techné ein Handlungswissen umfasst, und auch im heutigen Sprachgebrauch diese Bedeutung weiterhin aktuell ist, werden im vorliegenden Text unter ,Technik‘ zugunsten der Begriffsklarheit ausschließlich technische Apparate verstanden.

3. Vgl. Roland Innerhofer, Deutsche Science Fiction 1870-1914. Rekonstruktion und Analyse der Anfänge einer Gattung, Wien/ Köln/ Weimar, Böhlau, 2000, S. 15.

4. Vgl. Anne Seitz, „Zukunft schreiben. Prognostische Wissensfiguren in der fiktionalen Literatur des 20. Jahrhunderts“, in Heinrich Hartmann, Jakob Vogel (Hrsg.), Zukunftswissen. Prognosen in Wirtschaft, Politik und Gesellschaft seit 1900, Frankfurt a.M./ New York, Campus Verlag, 2010, S. 252 .

5. Vgl. Karl Riha, „Science fiction und Phantastik. Zur unterschiedlichen literarischen Reaktion auf den technischen Prozeß um die Jahrhundertwende“, in Götz Großklaus, Eberhard Lämmert (Hrsg.), Literatur in einer industriellen Kultur, Stuttgart, Cotta'sche Buchhandlung, 1989, S. 241. 
Kulturwissenschaft nicht unbemerkt, wobei besonders die Futurologie und Wissenspoetologie als neueste Ansätze die aktuelle Forschung prägen. ${ }^{6}$ Durch die science studies etablierte sich zudem die heuristische Annahme, dass Fakten den Erzählungen nicht zwangsläufıg vorausgehen und besonders soziale Tatsachen vielfach erst durch Narrative geschaffen werden. ${ }^{7}$ Vor der Frage, inwiefern literarische Narrationen an der Erfindung, Entwicklung und Existenz technischer Geräte - oder: materieller ,Tatsachen“ - teilnehmen, scheute sich die Literaturwissenschaft jedoch. Vorliegender Beitrag will anhand eines konkreten Beispiels diese Frage stellen.

\section{Literarische Figuration und technische Materialität}

Gegenstand der Betrachtung ist ein unscheinbarer Apparat, der aber erlaubt, die Vermittlung zwischen Literatur und Technik paradigmatisch aufzufächern. In der zweiten Hälfte des 19. Jahrhunderts taucht scheinbar unmotiviert die Vorstellung einer technisch-ästhetischen Kontrolle der bislang als anarchisch geltenden Düfte auf. Die auffälligste und am häufigsten genannte Maschine dieser Bemächtigung des flüchtigen, widerspenstigen, aber sehr affektiven Materials ist die ,Duftorgel“, die zwischen 1871 und 1932 in verschiedenen Werken der europäischen Literatur zu finden ist. Obwohl bereits im 17. Jahrhundert im Kontext des Farbklaviers projektiert, ${ }^{8}$ scheint die Duftorgel eine ,literarische Erfindung zu sein. Angesichts dieser Ausgangslage könnte sie als eine literarische Figuration verstanden werden, deren Auftauchen eine bestimmte diskursive, zeitlich begrenzte Formation zur Bedingung der Möglichkeit hatte.

Finden bereits in der Literatur um 1900 zwischen Olfaktion, Technik und Literatur zahlreiche Wechselwirkungen statt, wird die Komplexität im Zusammenspiel von Materialität und Narration in der Geschichte der ,Duftorgel jedoch in jenem Moment potenziert, in dem sich die literarische Figuration in einem technischen Objekt realisiert: Gut 130 Jahre nach dem ersten Auftauchen in fiktiven Texten baut 1996 der österreichische Künstler Wolfgang Georgsdorf die erste analoge Duftorgel. 2012 folgte der digitale Nachfolger namens Smeller 2.0. Dieses Instrument drängt die Frage auf, welche Bedeutung fiktive Erfindungen für den handfesten Bau technischer Geräte haben. Ist zu guter Letzt die eigentliche technische Innovation literarisch oder bildet sie zumindest eine genetische Linie mit der Literatur?

6. Untern den zahlreichen Publikationen vgl. exemplarisch Theisohn, Die kommende Dichtung sowie Joseph Vogl, „Einleitung“, in ders. (Hrsg.), Poetologie des Wissens um 1800, München, Wilhelm Fink, 1999, S. 9-16.

7. Vgl. z.B. Albrecht Koschorke, Wahrheit und Erfindung. Grundzüge einer Allgemeinen Erzähltheorie, Frankfurt a.M., Fischer, 2017, bes. S. 19-25.

8. Vgl. Jörg Jewanski, „Von der Farbe-Ton-Beziehung zur Farblichtmusik“, in ders., Natalia Sidler (Hrsg.), Farbe - Licht - Musik. Synästhesie und Farblichtmusik, Bern/ New York, Peter Lang, 2006, S. 149. 
Dieser Beitrag unternimmt also eine Invertierung der herkömmlichen Perspektive: Anstatt von der Übertragung von Technik in die Literatur wird die Wirkung fiktiver Texte auf ein technisches Objekt untersucht. Dazu wird in vier Schritten vorgegangen. Zunächst wird die literarische Figuration ,Duftorgel als diskursives Ereignis analysiert und ihre narrative Inszenierung dargestellt. Der zweite Teil des Aufsatzes wirft eine methodische Frage auf: Inwiefern kann Literatur technisch sein? In diesem Zusammenhang wird auf Gilberts Simondons Konzepte des technischen Objekts und der Erfindung zurückgegriffen. Die literarische Figuration und Simondons Technikphilosophie bilden die Grundlage, um im dritten Teil den erfinderischen Gehalt der literarischen Figuration herauszuarbeiten. Zum Abschluss wird die Narration als Existenzbedingung des technischen Objekts Smeller 2.0 bestimmt, in der die Grammatiken von Musik und Literatur das Material im doppelten Sinne formt.

\section{Bändigung eines widerspenstigen Sinns: Figurationen der Duftorgel um 1900}

Literarische Duftorgeln oder ähnliche technische Vorrichtungen finden sich z.B. in Kurd Laßwitz science fiction-Erzählung Bis zum Nullpunkt des Seins von 1871, Joris-Karl Huysmans Epochenroman $\dot{A}$ rebours von 1891, Paul Scheerbarts phantastischem Roman Münchhausen und Clarissa von 1906, in Christian Morgensterns grotesken Gedichten Die Geruchs-Orgel und Der Aromat von 1910 oder Aldous Huxleys dystopischen Roman Schöne neue Welt (engl. Orig. A Brave New World) von 1932. In jedem dieser literarischen Texte wird das flüchtige Material technisch unter Kontrolle gebracht und dessen affektive Wirkung auf den Riechenden zu ästhetischen, teilweise auch biopolitischen Zwecken eingesetzt.

Dass die technische Bändigung der Olfaktion gerade in der breit gefassten Jahrhundertwende in literarischen Texten, die gerade nicht einem naturalistischen oder realistischen Schreiben verpflichtet sind, virulent wird, erklärt sich aus dem historischen Kontext. Einerseits hielten im Zuge der Industrialisierung und Urbanisierung in der zweiten Hälfte des 19. Jahrhunderts mechanische Maschinen als eigene Existenzweisen Einzug in breitere Kreise der europäischen Gesellschaften. ${ }^{9}$ Andererseits handelt es sich bei der Olfaktion um jenen Sinn, dessen Funktionsweise zum Ende des 19. Jahrhunderts - und weit darüber hinaus - der Physiologie und Psychologie die größten Schwierigkeiten bereitete. ${ }^{10}$ Eine Apparatur wie der Duftorgel gelingt in der phantastischen oder

9. Vgl. Harro Segeberg, Literarische Technik-Bilder. Studien zum Verhältnis von Technik- und Literaturgeschichte im 19. und frühen 20. Jahrhundert, Tübingen, Niemeyer, 1987, S. 1-7.

10. Erst zum Ende des 20. Jahrhunderts klärten Richard Axel und Linda B. Buck die grundlegenden Vorgänge in der olfaktorischen Organisation, wofür sie 2004 den Nobelpreis für Medizin erhielten. 
in der Zukunft spielenden Fiktion die Beherrschung jenes Organs, das sich der wissenschaftlichen Forschung widersetzt. ${ }^{11}$

Den Anspruch auf Regulierung und Kontrolle reflektieren die meisten der literarischen Beispiele. ${ }^{12}$ In der Gesamtanlage der Texte besitzen allerdings die Duftorgel und die olfaktorische Erfahrung unterschiedliches Gewicht, das von einer illustrativen Bedeutung bei Huysmans und Huxley bis zu einer die Handlung strukturierenden Funktion bei Laßwitz reicht. Symptomatisch ist, dass in allen genannten Werken die Wirkung sowie die Komposition von technisch mediatisierter Duftkunst gleich erzählt werden: Die olfaktorische Wahrnehmung affektiert das ganze psychophysische System, stimuliert unwillkürlich Fantasie sowie Erinnerung und verhindert rationales Denken. Die Figurationen der Duftorgel schreiben offensichtlich die Dialektik zwischen Einbildungskraft und rationaler Kontrolle weiter, die Ästhetik und Pädagogik seit dem 18. Jahrhundert gleichermaßen beschäftigen. ${ }^{13}$

\section{Die Dichtung des Instruments: Erzählen in musikalischer Grammatik}

In den medialen Ordnungen, die die fiktiven Narrationen entwerfen, sind die Duftkunst und die Dichtung durchwegs gleichrangig: Wie die Literatur sind olfaktorische Kompositionen Teil der Künste. Diese Nobilitierung geschieht nicht nur beiläufig, wie z.B. in Huxleys Dystopie, wo alle Künste nur Regulierungsund Steuerungsfunktion besitzen. Die Geschichte der Ododik bei Laßwitz ${ }^{14}$ oder auch der konsequente Vergleich der Parfümkomposition mit der Literatur und der Musik bei Huysmans ${ }^{15}$ deuten auf eine strategische Neupositionierung der Olfaktion in der Ordnung der Sinne bzw. Künste, was sich auch an weiteren Texten verfolgen lässt. ${ }^{16}$

Vom poetologischen Standpunkt aus stellt sich allerdings die Frage, ob diese Herausstellung der affektiven und phantastischen Wirkung der Duftstoffe

11. Vgl. Helmut Berghoff, „Dem Ziele der Menschheit entgegen. Die Verheißungen der Technik an der Wende zum 20. Jahrhundert“, in Ute Frevert (Hrsg.), Das neue Jahrhundert. Europäische Zeitdiagnosen und Zukunftsentwürfe um 1900, Göttingen, Vandenhoeck \& Ruprecht, 2000, S. 61.

12. Besonders explizit reflektiert Laßwitz’ Erzählung Bis zum Nullpunkt des Seins (vgl. Kurd Laßwitz, Bis zum Nullpunkt des Seins. Erzählung aus dem Jahre 2371, Paderborn, Tredition, 2011 [1871], S. 9) und Aldous Huxleys A New Brave World diesen regulativen Anspruch.

13. Vgl. z.B. Anja Lemke, „Ästhetische Erziehung als Arbeit am Selbst. Schillers Bildungsprogramm aus der Perspektive postmoderner Kontrollgesellschaften“, in Thomas Glaser, Bettine Menke (Hrsg.), Experimentalanordnungen der Bildung, München, Wilhelm Fink, 2014, S. 127-141.

14. Laßwitz, Bis zum Nullpunkt des Seins, S. 9f.

15. Joris-Karl Huysmans, Ȧ rebours, in CEuvres complètes, Bd. 7, Genève, Slatkine, 1972 [1884], S. 177.

16. Vgl. z.B. Charles Baudelaire, Les Fleurs du mal, in ders., Euvres complètes, Bd. 1, Paris, Gallimard, 1975 [1861], S. 47-48; Theophile Thoré, „L’Art des parfums“, Ariel. Journal du monde élégant 13, 1836; H. G. Ludwigs, „Physiologische Lyrik“, Moderne Rundschau 9, 1891, S. 346. 
bzw. ihrer technischen Vermittlung zu einem spezifisch olfaktorischen Erzählen führt. Zunächst gilt es festzuhalten, dass sich die Passagen, in denen das Spiel und die Wirkung der Duftorgel erzählt wird, in allen Texten gleichen, wie die Gegenüberstellung einiger Passagen illustriert:

Springauf, Flieder und Rosen führten die Träume in die holde Zeit des Sommers und der jungen Liebe, [...] aber allmählich verschwimmen diese Düfte, wir glauben vor verwelkten Blumen zu stehen, und ein Gemisch von Jasmin und Schnittlauch durchzieht das Gemüt mit unendlicher Wehmut. ${ }^{17}$

\author{
Diese [Nieswurz-Sonate, SR] beginnt mit Alpenkräuter-Triolen \\ und erfreut sich durch eine Akazien-Arie. \\ Doch im Scherzo, plötzlich und unerwartet \\ zwischen Tuberosen und Eukalyptus \\ folgen die drei berühmten Nieswurz-Stellen [...]. ${ }^{18}$
}

Die Duftorgel setzt zu einem wunderbar belebenden Herbalcarpaccio an: Auf plätschernden Arpeggios aus Thymian, Lavendel, Rosmarin, Myrte, Estragon folgte eine kühne Scheinmodulation über die Gewürztonarten bis zu Ambra und dann langsam vagierend zurück über Sandelholz, Kampfer, Wacholder und Wiesenheu (mit subtilen Dissonanzen: einem Hauch Nierenpudding, einer zarten Andeutung Schweinekot) bis zur elegischen Wiederaufnahme der schlichten Aromatik des Auftakts. ${ }^{19}$

Die Materialität der Duftstoffe wird in den genannten Beispieltexten ausnahmslos durch drei Merkmale in Text übersetzt: ihre physische Quelle, den Kontext der olfaktorischen Erfahrung und ihre emotionale Wirkung. Eine eigene Syntax entwickelt das Riechen nicht. Im Gegensatz zu verwandten literarischen Texten aus der gleichen Zeit, die sich an einer ,olfaktorischen' Erzählung versuchen, ${ }^{20}$ formuliert die Figuration der Duftorgel keinen poetologischen Auftrag. ${ }^{21}$

Dass die Literatur der Duftorgel selbst nicht ,duftend“ wird, offenbart sich auch an der Eingliederung der olfaktorischen Künste in ein musikalisches Erzählschema bzw. Grammatik. Während die Stofflichkeit der Düfte und Gerüche über ihre Quelle oder dem Kontext von deren olfaktorischer Wahrnehmung erzählt wird, liefert die Musik die erzählerischen Schemata, in denen sich die literarische Duftkunst manifestieren kann. In den Texten von Laßwitz, Huysmans, Morgenstern und Huxley prägen diese gleichermaßen die Erzählung von Spiel und Rezeption eines Duftstückes ${ }^{22}$, seines institutionellen Rahmens

17. Laßwitz, Bis zum Nullpunkt des Seins, S. 10.

18. Christian Morgenstern, „Die Geruchs-Orgel“, in ders., Gesammelte Werke, Bd. 1: Galgenlieder. Palmström, Weyarn, Seehammer Verlag, 1998 [1910], S. 161.

19. Aldous Huxley, Neue schöne Welt [engl. Orig. A Brave New World], Frankfurt a.M., Fischer, 2015 [1932], S. 190f.

20. Literarische Texte können durchaus anhand von Düften und Gerüchen ihre eigene Funktionsweise: Vgl. Sergej Rickenbacher, „Duft und Text. Konstitution und Kritik olfaktorischer Eigenzeit bei Baudelaire und Huysmans“, in Stefanie Heine, Sandro Zanetti (Hrsg.), Transaktualität. Ästhetische Dauerhaftigkeit und Flüchtigkeit, Paderborn, Wilhelm Fink, 2017, S. 219-240 sowie ders., Wissen um Stimmung. Diskurs und Poetik in Robert Musils „Die Verwirrungen des Zöglings Törleß“ und „Vereinigungen“, Paderborn, Wilhelm Fink, 2015, bes. S. 237-290.

21. Einzig Christian Morgensterns groteskes Gedicht Die „Geruchs-Orgel“ bemüht die Olfaktion zur Inszenierung der Materialität von Sprache.

22. Die Ausnahme bildet Scheerbart, der die Architektur mit der Parfümerie und der Lüftungstechnik verbindet. Vgl. Paul Scheerbart, Münchhausen und Clarissa. Ein Berliner Roman, in ders., Das 
und des Instruments. „[D]ie Gesetze der Harmonie und Disharmonie“23 von Gerüchen erlauben die Komposition. Musikalische Termini wie Akkord, Triole, Synkope und Arpeggio charakterisieren das olfaktorische Spiel, die Stücke sind in Arien unterteilt oder als Sonaten klassifiziert. Es gibt „Gewürztonarten“ und selbst Duftgeschlechter, Dur- und „Mollgeruch“24. Angesichts der Leerstelle des Riechens in der Literatur bietet offensichtlich die Systematik der Musik eine fiktive innere Organisation an, in der die Olfaktion in eine Dynamik der Erzählung übersetzt werden kann.

\title{
Technik der Duftorgel zwischen Narration und Materialität
}

Die Materialität der Düfte formuliert sich also in einer musikalischen Grammatik und wird mittels entsprechender Narrative mediatisiert. Ausgespart blieb bislang die technische Seite der Figuration. Das fiktive technische Objekt nimmt nicht in allen Texten gleich viel Platz in Anspruch. Ausführlicher wird die Funktionsweise des Ododions einzig in Bis zum Nullpunkt des Seins ausgeführt - und die Duftorgel zudem mit einer historischen Genese versehen:

\begin{abstract}
Das Ododion (von o $\varpi \delta \eta$, der Geruch) oder Geruchsklavier wurde im Jahre 2094 von einem Italiener namens Odorato erfunden und im Laufe der Zeit, entsprechend den Fortschritten der Chemie bedeutend vervollkommnet. Das Instrument unserer Künstlerin war aus einer deutschen Fabrik und zeichnete sich durch seinen großen Umfang an Gerüchen aus [...]. Jeder Druck auf eine Taste öffnete einen entsprechenden Gasometer, und künstliche mechanische Vorrichtungen sorgten für die Dämpfung, Ausbreitung und Zusammenwirkung der Düfte. ${ }^{25}$
\end{abstract}

Ganz im Sinne einer herkömmlichen Kirchenorgel funktioniert das Ododion mechanisch. Das mechanische Grundprinzip dieser fiktiven Maschine scheint einfach zu sein. Die Differenz zur tatsächlich existierenden Orgel liegt zum einen im Einbau der Gasbehälter sowie ihrer Verbindung mit dem Luftkreislauf und zum anderen in der Ersetzung der tonerzeugenden Pfeifen durch stille Ausgänge. Der Text suggeriert auch, dass die Materialisierung der Duftorgel weniger von der Mechanik der Orgel als von den Fortschritten der Chemie abhängt, die für die Breite und wohl auch Erschwinglichkeit des Duftmaterials zuständig wäre.

Gerade die materiellen Eigenschaften von Duftmolekülen stellen aber die Funktionalität des Laßwitz'schen Instruments in Frage. Die nur kurz erwähnten „künstlich[n] mechanische[n] Vorrichtungen“, die Düfte gleich Tönen dämpfen, zu Akkorden mischen und im Raum verteilen, können nicht in gleicher Weise wie in der musikalischen Orgel funktionieren. Duftmoleküle fusionieren und verklingen nicht wie Schallwellen. Die Gleichzeitigkeit und die Mischung der unterschiedlichsten Gerüche im Raum würden ein zeitlich organisiertes Spiel

große Licht. Gesammelte Münchhausiaden, Frankfurt a.M., Suhrkamp, 1987 [1906], S. 30-32.

23. Laßwitz, Bis zum Nullpunkt des Seins, S. 9.

24. Ibid., S. 12.

25. Laßwitz, Ibid., S. $9 f$. 
verhindern. Ein ausgeklügeltes Lüftungssystem ist daher notwendig, um eine Duftorgel spielen zu können.

Dass die Konstruktion einer geeigneten Architektur Bedingung der Existenz ist, scheinen Bis zum Nullpunkt des Seins und besonders Münchhausen und Clarissa zu ahnen. Das Laßwitz'sche Ododion ist nur für den Hausgebrauch konzipiert. Die großen Duftorgel-Konzerte dagegen finden in einem eigens gebauten Odoratorium: „E]s [lag] in der Natur der Ododik, dass die Odoratorien nur für eine verhältnismäßig geringe Zahl von Zuriechern gebaut werden konnte. “ ${ }^{26}$ Wird die spezielle Räumlichkeit des Odoratorium bei Laßwitz nur angedeutet, entspricht Scheerbarts Duftorgel einem ganzen Palast. Sogenannte Schwarzkünstler bewohnen nach dem Bericht von Baron Münchhausen in Australien ein Haus, dessen Sauerstoffzufuhr nicht über die Fenster, sondern über ein Lüftungssystem verläuft. ${ }^{27}$ Dieses ist auch die technische Bedingung für die Experimente der Schwarzkünstler mit „Gerüche[n] und Luftkompositionen“" ${ }^{28}$, die sie mittels der Lüftung verteilen und verschwinden lassen. Die literarische Figuration der Duftorgel um 1900 formuliert in nuce eigentlich genau jenes technische Problem aus, an dem sowohl das Riechkino ${ }^{29}$ als auch die Realisierung der Duftorgel lange scheiterte: Die Notwendigkeit eines konstanten, exakt steuerbaren Lüftungssystems, das überhaupt erst die Erfahrung von zeitlich organisierten Duftkompositionen ermöglicht.

Die Figuration der Duftorgel zeugt also, so kann zusammengefasst werden, von einer Vielzahl verschiedener Vermittlungen. Die Düfte werden als affektiv wirksame Stoffe inszeniert, die technisch und ästhetisch kontrolliert werden, um ihre Wirkung auf Körper und Geist der Menschen zu regulieren. Die Grammatik, in der sowohl die Duftkunst narrativ zu Entfaltung kommt als auch das technische Objekt inszeniert wird, ist die Musik. Gleichzeitig antizipiert die literarische Figuration die entscheidenden technischen Fragen, die es für eine Materialisierung der Duftorgel zu lösen gilt: die Steuerung der Diffusion und den architektonischen Raum.

In der Fiktion ist diese Erfindung also durchaus technisch. Aber ist das technische Objekt auch literarisch? Für die Frage, ob von der Literatur in die Technik ebenfalls eine Übersetzung stattgefunden hat, wird im nächsten Abschnitt auf Simondons Begriff der Technizität und sein Konzept der Erfındung eingegangen, mit denen die literarische Existenzweise der Duftorgel mit ihrer technischen gekoppelt und als genetische Linie bestimmt werden kann.

26. Ibid., S. 29.

27. Vgl. Scheerbart, Münchhausen und Clarissa, S. 31.

28. Vgl. ibid.

29. Vgl. Ludger Kaczmarek, Odorated Talking Pictures (OTP), [http://filmlexikon.uni-kiel.de/index. php?action= lexikonEttag=detEtid=1092], Stand: 24. August 2017. 


\section{Essenz und Erfindung technischer Existenzweisen bei Simondon}

In Die Existenzweise technischer Objekte entwirft Simondon eine Philosophie der Technik, die sowohl ontologischen als auch genealogischen Gehalt besitzt. Die Essenz eines technischen Objekts macht nach Simondon nicht das materielle Vorhandensein aus. Vielmehr konstituiert es eine Technizität, die transsubjektiv sowie -objektiv bestimmt ist. ${ }^{30}$ Sie ist eine Virtualität, die durch die Existenz von technischen Objekten manifest wird, sich jedoch nicht vollständig in ihnen artikuliert. Durch diese Vorgängigkeit und den Überschuss sind diese technische Existenzweisen grundsätzlich offen für weitere Entwicklungen - in Simondons Terminologie: die Konkretion -, wodurch sich genetische Linien entwickeln können. ${ }^{31}$ Diese neuen Linien besitzen vorerst keine kausale Finalität ${ }^{32}$ und sind nicht mit schlichten Weiterentwicklungen oder Innovationen gleichzusetzen. Vielmehr handelt es sich um Diskontinuitäten, durch die neue funktionale und strukturale Wirklichkeiten gekoppelt werden.

Allerdings sind nicht technische Objekte erfinderisch. Weil ihnen die Fähigkeit zum kreativen Handeln fehlt, können sie nicht selbst die Virtualität der Technizität in materiellen Prozessen aktualisieren. ${ }^{33}$ Vorwiegend der Mensch besitze diese Eigenschaft dank einer prospektiven schöpferischen Intuition in Form eines symbolischen Denkens:

\footnotetext{
Die Erfindung, die Schöpfung des Individuums ist, setzt beim Erfinder die intuitive Kenntnis der Technizität der Elemente voraus; die Erfındung vollzieht sich auf dieser Zwischenebene zwischen Konkretem und Abstraktem [...] und setzt dabei die vorgängige Existenz und die Kohärenz der Vorstellungen voraus, welche die Technizität des Objekts mit Symbolen abdecken, die Teil einer Systematik und einer imaginativen Dynamik sind. ${ }^{34}$
}

Die Erfindung als kreative Geste ist immer in die Zukunft ausgreifend. ${ }^{35}$ Entscheidend für die Fähigkeit der Menschen, einen solchen Sprung zu initiieren, ist neben der schöpferischen Intuition das symbolische und schematische Denken. Fasst es Simondon in Die Existenzweise technischer Objekte noch als Leistung 'eines' menschlichen Individuums, integriert er dieses Vermögen in seiner Vorlesung Invention et imagination in einen sozialen und historischen Rahmen.

30. Gilbert Simondon, Die Existenzweise technischer Objekte [franz. Orig. Du mode d'existence des objets techniques], Zürich, diaphanes, 2012 [1958], S. 152.

31. Jean-Hugues Barthélémy, „Simondon - Ein Denken der Technik im Dialog mit der Kybernetik“, in Erich Hörl (Hrsg.), Die technologische Bedingung. Beiträge zur Beschreibung der technischen Welt, Frankfurt a.M., Suhrkamp, 2011, S. 96-104 sowie Johannes Schick, „Die Erfindung der Offenheit. Kreatives Handeln im Ausgang von Gilbert Simondon“, in ders., Roberto Hugo Ziegler (Hrsg.), Die innere Logik der Kreativität, Würzburg, Königshausen \& Neumann, 2013, S. 153-179.

32. Vgl. ibid., S. 289.

33. Die Absolutheit dieser These ist angesichts der gegenwärtigen Informationstechnologie natürlich zu relativieren.

34. Simondon, Existenzweise technischer Objekte, S. 54, Hervorhebung SR.

35. Ibid., S. 57. 
Die Erfindung eines Einzelnen resultiert aus der Saturation eines symbolischen Systems, das dem Individuum genau zu dieser Vermittlung dient:

De l'univers de symboles intérieurement organisé, tendant à la saturation, peut surgir l'invention, qui est la mise en jeu d'un système dimensionnel plus puissant, capable d'intégrer plus d'images complètes selon le mode de la compatibilité synergique. ${ }^{36}$

$\mathrm{Zu}$ erfinden ist also nach Simondon ein explizit kulturell bedingter Vorgang ${ }^{37}$, der immer auch einen Wandel in der größeren „Ordnung“ indiziert ${ }^{38}$ und die Grundlage für weitere Entwicklungen schafft. Angesichts der Vermittlung zwischen Mensch und Technik kann auch die Sprache, mithin die Literatur als eine solche symbolische Ordnung bestimmt werden.

Auf diese zahlreichen Mediationen zwischen Mensch, technischem Objekt und nicht-menschlicher Natur zielt Simondons Technikphilosophie trotz der ontologischen Bestimmung der Technik grundsätzlich ab. ${ }^{39}$ Die Übersetzungen zwischen den Milieus sind jedoch auch der Grund dafür, dass die Aktualisierung einer Technizität noch nicht deren dauerhafte Existenz garantiert. Erfindungen unterliegen Latenzzeiten und Regressionen. ${ }^{40}$ Der Fortbestand hängt von zwei verschiedenen Bedingungen ab. Erstens muss der symbolisch organisierte Erfindungsprozess in eine interne Funktion ,und ' in eine externe strukturale Kopplung münden, wie die Dampfmaschine erst durch und mit dem Bergbau die industrielle Revolution dominieren konnte. Zweitens muss ein technisches Objekt mit seiner Außenwelt, zu der auch die Gesellschaft als kulturelle Organisationsform gehört, kompatibel sein. So konnten sich z.B. alternative Treibstoffe für Motoren bisher nicht durchsetzen, obwohl technische Lösungen seit Jahrzehnten vorhanden sind.

Angesichts der zwei Bedingungen der Existenz von Erfindungen definiert Simondon drei verschiedene Schichten im einzelnen technischen Objekt. ${ }^{41}$ Den Kern des technischen Objekts bildet die Materialisierung der Technizität. Es handelt sich um die technische Schicht, die den meisten Nutzern zwar unbekannt sei, jedoch die eigentliche Erfındung ausmache. Die mittlere Schicht ist „mi-technique et mi-langage “42 und garantiert die externe Kommunikation zwischen technischen Objekten und ihren Benutzern (z.B. bei Motoren mit Angaben zu Zylinder oder Drehzahlen, bei Waschmaschinen zu Füllmenge und Stromanschluss etc.). Gleichzeitig durchzieht die Wirklichkeit der verschiedenen technischen Objekte ebenfalls ein Kommunikationsnetz, das die verschiedenen Objekte dieses Bereichs miteinander verbindet (z.B. der Gebrauch des Aluminiums bei Flugzeugen, Autos, Möbeln oder Haushaltsgeräten in den 1960er Jahren oder die heutige Verwendung von Spiegelglas, das gleichermaßen auf Handys wie auf

\footnotetext{
36. Simondon, L'invention dans les techniques, S. 275.

37. Vgl. ibid., S. 293.

38. Vgl. ibid., S. 296.

39. Vgl. ibid., S. 53, S. 293-296.

40. Vgl. ibid., S. 281 und S. 285.

41. Vgl. ibid., S. $282 f$.

42. Vgl. ibid., S. 282.
} 
Backöfen zu finden ist). Nur am Rande ,technisch` ist die äußerste Schicht eines Objekts. Gemeint sind Formen und Gebrauchsweisen, die sich nicht aus dem technischen Wesen des Objekts ableiten - wie z.B. die Vorliebe für bestimmte Farben, Designs oder der Gebrauch als Statussymbol - und gegenüber der Technizität bestenfalls neutral, meist aber verstellend und störend sind. ${ }^{43}$

Fraglich ist, ob Simondon die Bedeutung der verbalen und non-verbalen Diskursivierung von technischen Objekten im Akt der Erfındung nicht unterschätzt, da sie unter bestimmten Bedingungen genetische Sprünge initiieren kann und sicherlich wesentlich zur Stabilität technischer Objekte beiträgt. ${ }^{44}$ Gerade Narrative verfügen über die Eigenschaft, Kompatibilitäten herzustellen und in der Folge zu stabilisieren. Zwar erlangt das technische Objekt nach Simondon im Verlaufe seiner Konkretion eine Offenheit, d.h. immer mehr Möglichkeiten zu neuen Kopplungen, die mit einer zunehmenden Ähnlichkeit mit Organismen und somit einer Naturalisierung gleichgesetzt werden kann. ${ }^{45}$ Die meisten technischen Objekte existieren jedoch in einem künstlichen Milieu im Sinne eines ,Labors: „Die artifizialisierte Pflanze kann nur in jenem Pflanzenlabor existieren, welches das Gewächshaus mit seinem komplexen System thermischer und hydraulischer Regulationen darstellt." ${ }^{46}$ Der Mensch agiert dort als Koordinator der Maschinen und als Mediator zwischen Maschine und Umwelt. Um diese Funktionen ausüben zu können, sind jedoch auch Narrative notwendig, die einer Erfindung ihre soziale - nicht technische - Existenzberechtigung verleihen. Damit ist der kreative Akt nicht allein der Entwurf einer technischen Lösung, sondern auch die Herstellung von innerer und äußerer Komptabilität, was den Akt der Erfındung dehnt und vom Individuum löst.

\section{Fiktive Duftorgeln als Erfindungen?}

Eindeutig ist hinsichtlich der fiktiven Duftorgeln, dass sie keine technischen Objekte sind, in denen sich eine Technizität in einem Instrument materialisiert. Gleichwohl können die Erzählungen von der technischen Bändigung und Steuerung der widerspenstigen Olfaktion durchaus als Etappen in einem erfinderischen Akt klassifiziert werden, der nicht als Leistung eines Individuums, sondern als gedehnter genetischer Sprung aus einem saturierten Symbolsystem verstanden wird. Diese Sichtweise positioniert nicht alleine die erzählende Literatur als Medium der Erfindung, sondern erweitert auch Simondons Begriffe von Symbol und Schema um einen narrativen Zeichenbegriff. Die Literatur wird dabei nicht zu einem technischen Objekt, konzipiert und katalysiert dennoch die Vermittlungen in der inneren und äußeren Schicht des materialisierten Apparates.

43. Vgl. dazu Schick, Erfindung der Offenheit, S. 172f.

44. In gewissen Passagen lässt Simondon ein solches Bewusstsein doch auch aufblitzen. Vgl. Simondon, L'invention dans les techniques, S. 294.

45. Vgl. Barthélémy, Simondon - Ein Denken der Technik, S. 103.

46. Simondon, Existenzweise technischer Objekte, S. 43. 
Disziplinäre Ordnungen und übergreifende Dispositive erlauben Verbesserungen, Innovationen und sogar Erfindungen. Gleichzeitig verhindern sie die Aktualisierung gewisser anderer virtueller Technizitäten. Von diesem Gleichgewicht von Potenz und Latenz sowie dessen Überwindung zeugt gerade die Zukunftsliteratur um 1900. Die Autoren von phantastischen Erzählungen oder science fiction-Literatur - Autorinnen gab es kaum - waren oftmals selbst Ingenieure oder Naturwissenschaftler, mindestens aber technisch gut ausgebildet. ${ }^{47}$ Dies trifft größtenteils auch auf die hier besprochenen Autoren zu. ${ }^{48}$ Es ist bezeichnend, dass ihre Erfindungen vielfach ausschließlich ,literarisch sind. Gerade die Lösung aus der naturwissenschaftlichen Disziplin ermöglicht offensichtlich das Denken neuer technischer Objekte. Technische Erfindungen mögen ihre Aktualisierung in neuen funktionalen und strukturellen Kopplungen finden. Ihr geht aber häufıg der Wechsel in eine andere Disziplin voraus - im Fall der Duftorgel von der physikalischen Mechanik und der organischen Chemie in die Literatur. Es wird einem saturierten Symbolsystem entkommen, was die Gründung einer neuen genetischen Linie erlaubt.

Genauso entscheidend für die Existenz des erfundenen technischen Objekts ist jedoch die Vermittlung zwischen der äußeren Schicht des technischen Objekts mit der kulturellen Sphäre, wie gerade Wolfgang Georgsdorfs Smeller 2.0 und dem Osmodrama-Festival zeigt. Die literarische Figuration mit ihrer Grammatik der Musik ermöglicht nicht nur die Kopplung der Erfindung an eine Kultur, sondern wirkt gleichsam auf deren technische Funktion zurück.

\section{Narrative Formierung des technischen Objekts: Wolfgang Georgsdorfs Smeller 2.0}

Die erste digitale Duftorgel Smeller 2.0 ist keine creatio ex nihilo. Vielmehr lässt sich eine genetische Linie von der literarischen Figuration über das Riechkino bis zur tatsächlichen Duftorgel ziehen. Ihre Verbindung sind die technischen Herausforderungen, die vom Lexikon der Filmbegriffe 2012 exakt aufgelistet werden: „Die Achillesferse der signalkodierten Geruchsfilme ist und bleibt die technische Ausbringung und Entfernung (Lüftung, Klimaanlage, spezielle Düsen) sowie das Timing der gewünschten Düfte." ${ }^{49}$ Es werden also genau jene Hürden benannt, deren Überwindung Laßwitz' und Scheerbarts Erzählungen projektieren.

Wolfgang Georgsdorf - und sein Team bestehend aus Architekten, Parfümeuren, Mechatronikern, Ingenieuren, Chemikern und Lüftungstechnikern

47. Vgl. Dina Brandt, Der deutsche Zukunftsroman. Gattungstypologie und sozialgeschichtliche Verortung, Tübingen, Niemeyer, 2007, S. 42-50, sowie Riha, Science fiction und Phantastik, S. 242.

48. Laßwitz war promovierter Mathematiker und Physiker, Huxley wuchs in einer Familie von Wissenschaftlern auf und wollte zunächst Arzt werden, Scheerbart war zwar ausgebildeter Geisteswissenschaftler, versuchte sich aber als Erfinder eines perpetuum mobile.

49. Vgl. Kaczmarek, Odorated Talking Pictures (OTP). 
- dürfen getrost für sich in Anspruch nehmen, diese technischen Probleme mit dem Smeller 2.0 grundsätzlich gelöst zu haben. ${ }^{50}$ Die 1,6 Tonnen schwere Duftorgel, die 6,5m breit, 3,2m hoch und 2m tief ist, enthält aktuell 64 „Quellkammern“, die mit ebenso vielen synthetischen oder natürlichen Düften gefüllt werden können. Komplexe Düsen, die auch in Atomkraftwerken zum Einsatz kommen, erlauben nicht nur die präzise Einspeisung der Düfte in einen konstanten Luftstrom, sondern können auch ihre Diffusion formen. Jede Manipulation verändert die plastische Erscheinung des Geruchs. Angesteuert werden die Düsen entweder durch digital gespeicherte Kompositionen oder durch ein Interface, zumeist eine Klaviatur.

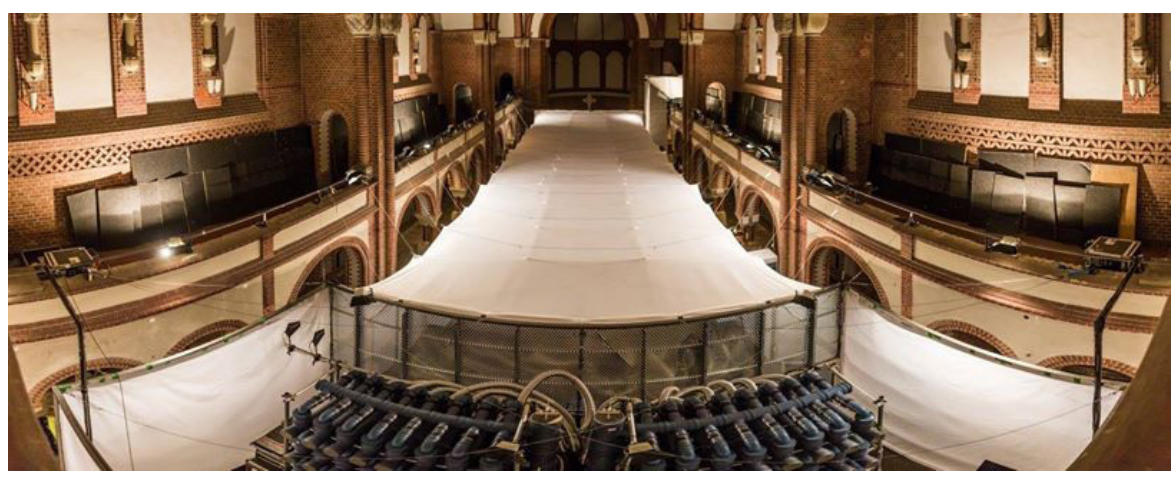

Fig. 1: Der Smeller 2.0 in der St. Johann Evangelist Kirche, Juli 2017, @ Gianmarco Bresadola

Die Duftstoffe treten dann aus dem sogenannten „Hauchmaul“ aus und verwirbeln sich im Raum. Ohne exakt diesen Raum - oder besser das architektonischtechnische Misch-Milieu, das Georgsdorf „Zuriecher“ nennt - würde die Duftorgel nicht funktionieren. Das längliche Zelt aus Gleitschirmstoff durchzieht nämlich ein gleichmäßiger Luftstrom, auf dem die Duftmoleküle durch den „Zuriecher“ getragen - und zuhinterst wieder abgesaugt werden. Das Luftvolumen wird so alle zwei Minuten komplett ausgetauscht und eine exakte Sequenzialisierung der Düfte möglich. Das technisch-architektonische Milieu des Smellers 2.0 zeigt nochmals unmissverständlich, wie abhängig die Existenz eines abstrakten technischen Objekts von seiner Umgebung ist, die in diesem Fall mit großem Aufwand artifiziell hergestellt werden muss.

Der Kunst ist genealogisch sowie terminologisch die Artifizialität immer schon eingeschrieben gewesen, weswegen dieser Befund nicht als Kritik an Georgsdorfs Osmodrama verstanden werden darf. Im Gegenteil, Georgsdorf kann für sich reklamieren, in der Duftorgel eine neue Technizität aktualisiert zu haben: Er ermöglichte mittels dem Einbau der High-Tech-Düsen das Funktionieren der Duftorgel, verbindet strukturell Chemie mit Mechanik und schafft ein

50. Über den Smeller 2.0 existiert bislang meines Wissens noch keine einschlägige wissenschaftliche Publikation. Zur Konstruktion und Funktion des Smeller 2.0 vgl. besonders [http://smeller.net/ about/], Stand: 24. August 2017. 
Misch-Milieu, in dem das technische Objekt existieren kann. Gleichwohl zeigt gerade sein Osmodrama-Festival die Fragilität dieser Existenz, die mehrheitlich kulturell, teilweise physiologisch bedingt ist.

Hinsichtlich des Zusammenhangs von Erfindung, Materialität und Narration sind folgende Faktoren von großer Bedeutung: Erstens kannte das feste Programm des Festivals neun verschiedene Stücke, von denen jedoch nur drei rein olfaktorische sind, wobei zwei erst im Verlauf des Festivals dazukamen. ${ }^{51}$ Alle anderen sind intermedial und synästhetisch, wobei Akustisches und Visuelles immer Bestandteil ist, Musik und Filme gelegentlich. Auch im Extraprogramm des Festivals findet sich keine weitere, rein olfaktorische Aufführung. Das Festival wird in Anlehnung an das Theater „Osmodrama“ getauft, der Untertitel lautet „Storytelling with scents“ und die einzelnen Kompositionen heißen „Synosmien“. Es werden also ähnliche narrative Strategien verfolgt, wie sie in Laßwitz' Am Nullpunkt des Seins oder Huxleys A New Brave World zu finden sind: Der Smeller 2.0 verbleibt weiterhin in einer musikalischen Grammatik, ergänzt durch einige Aspekte der Literatur und des Films. Zweitens ist die verbale und visuelle Narrativierung des technischen Objekts auffällig. Die technischen Elemente werden mit organischen Metaphern belegt bzw. naturalisiert: Die Gasbehälter werden zu „Quellkammern“, die Ansammlung von Rohrenden zum „Hauchmaul“ oder das Zelt zum „Zuriecher“. Für Simondon ist die Konkretion eines technischen Objekts die fortschreitende Naturalisierung, sprich die Angleichung an einen Organismus. ${ }^{52}$ Es scheint, als ob die Sprache die Autarkie des Smeller 2.0 vorbereiten soll. Drittens balanciert das Erscheinungsbild von Georgsdorfs Duftorgel geschickt zwischen Mythologie und Technik. Das „Hauchmaul“ erinnert an die Bewegungsdynamik einer mehrköpfigen Schlange oder eines Drachens, was mittels der ständig wechselnden Beleuchtung akzentuiert wird.

51. [Vgl. https://osmodrama.com/img/2016/06/Osmodrama_Programmheft_V11_RGB_engl_dt.pdf], Stand 24. August 2017.

52. Vgl. Barthélémy, Simondon - Ein Denken der Technik, S. 96-106 sowie Schick, Die Erfindung der Offenheit, S. 153-179. 


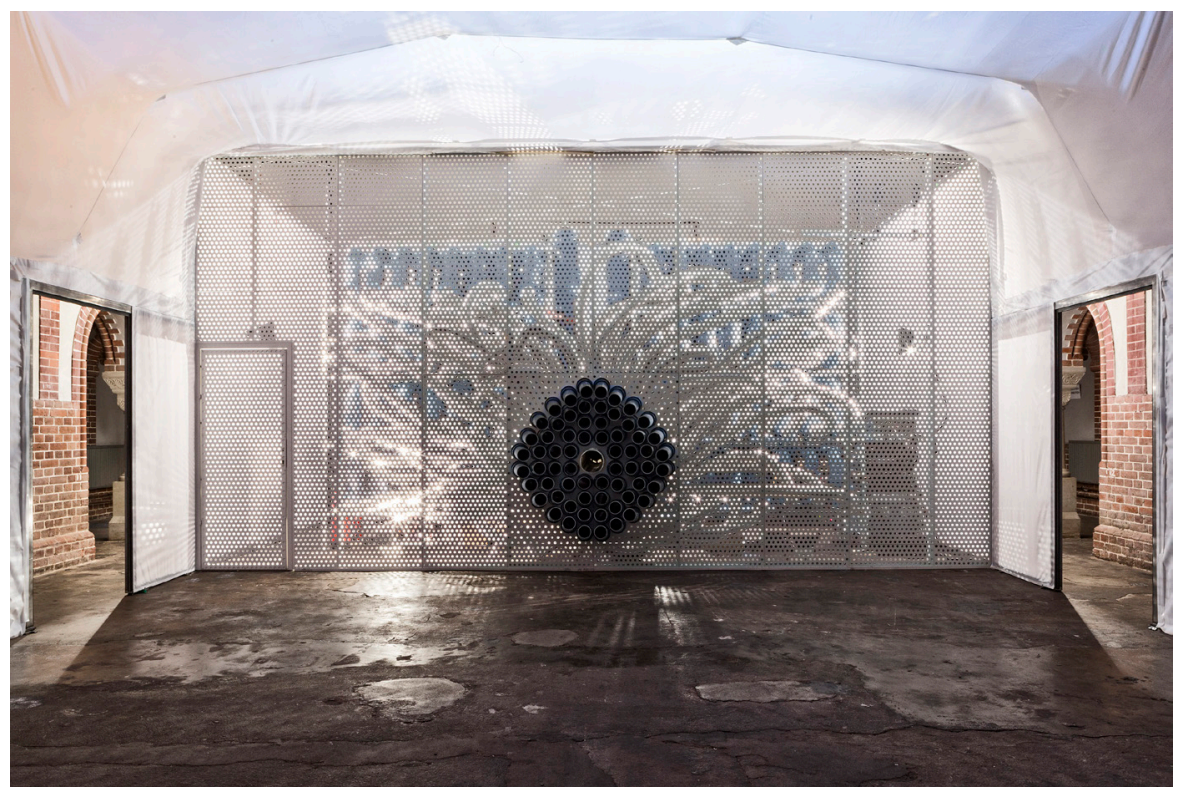

Fig. 2: Das Hauchmaul des Smeller 2.0, September 2017, @ Julian van Dieken

Diese Narrativisierung und Inszenierung des technischen Objekts setzt sich in zahlreichen Ausstellungsberichten und Interviews mit Beteiligten fort und nicht zuletzt etablierte Georgsdorf und sein Team eine intensive audiovisuelle Dokumentation des Festivals über die bekannten social media-Kanäle. ${ }^{53}$ Mit anderen Worten: Es wird ein großer erzählerischer Aufwand betrieben, um das technische Objekt Smeller 2.0 für Kopplungen mit der bestehenden Kultur offen und somit in Existenz zu halten.

Betrifft diese erzählerische Kopplung des technischen Objekts an eine Kultur vor allem die äußere Schicht, manifestiert sich die Übernahme der musikalischen und literarischen Grammatik im Instrument selbst. Nicht zufällig heißen die Duftkompositionen „Synosmien“ und „Osmodramen“. Diese Stücke orientieren sich an musikalischen Strukturen wie Melodien und Akkorden bzw. erzählerischen Sequenzen. Das Festival wirbt selbst mit einer neuen Form einer narrativen Kunst: „Osmodrama ist die Kunst des zeitbasierten Komponierens und Erzählens mittels des Smeller 2.0." ${ }^{54}$ Im Gegensatz zu den fiktiven Duftorgeln, in denen vorwiegend die Musik das Erzählschema von Duftkunst gebildet hat,

53. Vgl. die Homepages [http://georgsdorf.com/], Stand: 24. August 2017; [http://smeller.net/], Stand: 24. August 2017 sowie [https://osmodrama.com/], Stand: 24. August 2017; die FacebookSeite [https://de-de.facebook.com/smeller.net/], Stand: 24. August 2017; den Twitter-Account [https://twitter.com/osmodrama?lang=en], Stand: 24. August 2017; den Youtube-Kanal [https:// www.youtube.com/channel/UCQ79J-4URhViTz4f6Z3TMnA], Stand: 24. August 2017 sowie die zahlreichen Presseartikel und Interviews.

54. Vgl. [http://osmodrama.com/], Stand: 3. Oktober 2016: „Osmodrama is the art of timebased composing and storytelling via Smeller 2.0.“ 
scheint der Smeller 2.0 aber beide Grammatiken zu übernehmen. Die Literatur geht somit als zweites Erzählschema in das technische Objekt ein.

Die Musik bleibt aber im Vordergrund. Ihre Grammatik ist dominierend. Georgsdorf hat bereits früh ein an der Musik angelehntes Notationssystem entwickelt, mit dem er Geruchskompositionen entwirft. ${ }^{55}$ Auch dringt die Musik in die mittlere Schicht des Smeller 2.0 ein, in der die Kommunikation zwischen technischem Objekt und Benutzer stattfindet: Die Schnittstelle zwischen dem Duftkünstler Georgsdorf und seinem Instrument bildete bislang immer ein Musikinstrument, allen voran eine Keyboard-Klaviatur. Die Grammatik der Musik strukturiert also nicht nur die literarische Figuration, sondern koppelt auch das realisierte technische Objekt mit seinem Koordinator. Die narrative Kopplung zwischen Innen und Außen formt also die Materialität von dem technischen Objekt ,und“ der Duftkunst.

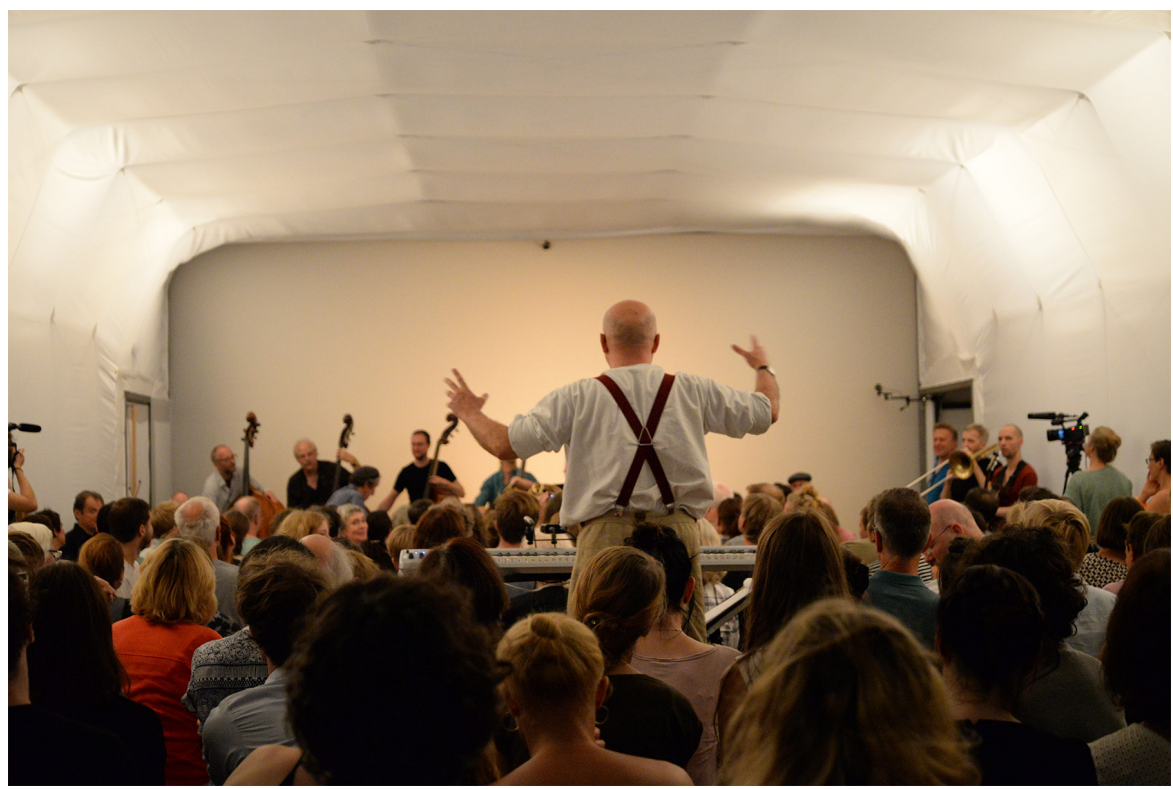

Fig. 3: Wolfgang Georgsdorf [Mitte] an einem akustisch-olfaktorischen Konzert, September 2017, () Adil Razali

Die spärlichen Erfahrungen, die bisher mit dem Smeller 2.0 gesammelt werden konnten ${ }^{56}$, werfen jedoch die Frage auf, wie stabil und beständig diese Kopplung von Duftorgel mit der Kultur über musikalische und literarische Grammatiken ist. Aus technischen Gründen besitzen die Stücke einen unvergleichlichen

55. Vgl. Andreas Egger, „Kunstparcours der Sinne. Sinnesrausch im OÖ Quartier in Linz“, kunst: art 281, 2012, S. 3 sowie [http://smeller.net/about/], Stand: 24. August 2017.

56. Bislang war der Smeller 2.0 erst drei Mal während weniger Wochen im Einsatz: 2012 an der Ausstellung Sinnesrausch im OK Linz, als eigenes Festival Osmodrama im Radialsystem V in Berlin im Sommer 2016 sowie dieses Jahr im Martin Gropius-Bau im Rahmen der Ausstellung Welt ohne Außen. 
Rhythmus, der von Entschleunigung geprägt ist. Unser Zeiterleben während des Riechens ist zudem nicht mit dem Sehen und Hören vergleichbar. Insofern scheinen „Synosmien“ und „Osmodramen“ auf eine eigenständige Formsprache zu drängen. Vielleicht erlaubt der Smeller 2.0 dereinst eine eigene olfaktorische Grammatik, einen „Duft“ der Duftwahrnehmung. Damit würde sich das Riechen als Sinneswahrnehmung erst richtig individuieren - genauso wie es nach Simondon die Photographie mit dem Sehen getan hat. ${ }^{57}$

57. Vgl. Schick, Die Erfindung der Offenheit, S. 169. 